\title{
X Congreso |lbérico de Agroingeniería

\section{Primer balance de agua, sales y nitrógeno en la cuenca de la Clamor Vieja, Castelflorite, Santalecina, Alcolea de Cinca (Huesca). Drenaje de un regadío modernizado}

\author{
R. Andrés ${ }^{1}$, L. Royes ${ }^{2}$, J.A Cuchí ${ }^{1}$ \\ 1 Escuela Politécnica Superior de Huesca. Carretera de Cuarte s/n. 22071-Huesca; raulandres@unizar.es, \\ cuchi@unizar.es \\ 2 Agrostock. Pol. Ind. Fraga Este, s/n. 22520 Fraga -Huesca; lourdesroyes@hotmail.com
}

\begin{abstract}
Resumen: Hay un creciente interés sobre la gestión de agua en las zonas regadas, riego y drenaje y es importante disponer datos concretos a diferentes escalas de cuenca. Se presenta un estudio preliminar de la Comunidad de Regantes San Pedro de Castelflorite (CRSPC) puesta en riego a pie hacia 1980 y modernizada a aspersión hacia 2001. Situada sobre un terreno fundamentalmente arcilloso, los regadíos de esta zona están afectados por graves problemas de salinidad que limitan el cultivo de amplias zonas. La salinidad de los horizontes superficiales, de origen geológico, es consecuencia de los grandes movimientos de tierra sin capaceo que se llevaron a cabo en su transformación inicial. La CRSPC drena, en parte al barranco de la Clamor Vieja, enclavado en los términos de Castelflorite, Santalecina y Alcolea de Cinca (Huesca) drena una cuenca de 11.450 ha, de las que aproximadamente la mitad están en riego con arroz, maíz, cebada y alfalfa. El presente trabajo es analizar el uso del agua y determinar la cantidad y calidad del drenaje mediante balance de agua, sales y nitrógeno en los años 2009 y 2010. El régimen hídrico de la cuenca está regulado esencialmente por el riego. Durante las campañas de riego el caudal de drenaje aumenta considerablemente siendo el volumen de agua drenado seis veces superior al de fuera de la campaña de riego.
\end{abstract}

Palabras clave: riego; drenaje; Aragón.

\section{Introducción}

El análisis por cuencas es uno de los métodos de realizar estudios de eficacia de regadíos y liberación de sales y nutrientes de un polígono de riego. Por ejemplo, se ha aplicado en la CR LASESA $[1,2,3]$.

El presente trabajo analiza la cuenca de la Clamor Vieja, un pequeño tributario del rio Cinca. La cuenca tiene una superficie de 11.450 ha. El 46\% es secano. En el regadío, la mayor parte corresponde a la CRSP que se puso en riego secuencialmente de 1972 a 1976 a pie. La nivelación de parcelas, y la geología arcillosa, condujo a importantes problemas de conductividad hidráulica y sodicidad [4]. Para combatirlo, se inició un proceso gradual y complejo de transformación a riego por aspersión.

El presente trabajo analiza el uso del agua y determina la cantidad y calidad del drenaje mediante balance de agua, sales y nitrógeno en los años 2009 y 2010. 


\section{CONGRESO IBÉRICO DE AGROINGENIERÍA \\ X CONGRESSO IBÉRICO DE AGROENGENHARIA \\ 3 - 6 septiembre 2019, Huesca - España}

\section{Localización}

La CRSP está situada en los términos de Alcolea de Cinca, Castelflorite, Peralta de Alcolea, San Miguel de Cinca, y Sariñena (Huesca, España). Se localiza en su margen derecha del tramo medio del río Cinca y gestiona una superficie de casi 5.000 ha de los sectores hidráulicos XVII, XVIII, XIX, XX y XXI del Canal del Cinca, acequia de Terreu en la Comunidad General de Riegos del Alto Aragón (Figura 1).

El barranco de la Clamor Vieja drena una cuenca de 11.450 ha, de las que aproximadamente el 54\% están en regadío. El $92 \%$ de este regadío pertenece a la CRSP y el 8\% restante a la Comunidad de Regantes de Santa Cruz. Los retornos de riego provienen esencialmente de la CRSP. En los años 70, esta zona se trasformó en regadío a pie y 30 años después se ha sustituido la red de distribución cambiando las acequias por tuberías a presión. A nivel de parcela también se ha cambiado mayoritariamente a riego por aspersión a excepción de la zona baja de los sectores XVII, XVIII, XIX donde aún predominaba, en el momento del estudio, el riego por superficie.

El clima se caracteriza por un período frío de cinco meses, otro cálido de tres: Llueve en primavera y otoño, con un periodo seco de 9 meses, de febrero a mediados de octubre, tormentas aparte. La precipitación media anual son $370 \mathrm{~mm}$. La evapotranspiración de referencia (ET0) $1132 \mathrm{~mm}$ al año. La humedad relativa media estuvo entre el 54\% de julio, y el $78 \%$ de diciembre 2009. La velocidad del viento osciló entre 1,2 y $2 \mathrm{~m} / \mathrm{s}$.
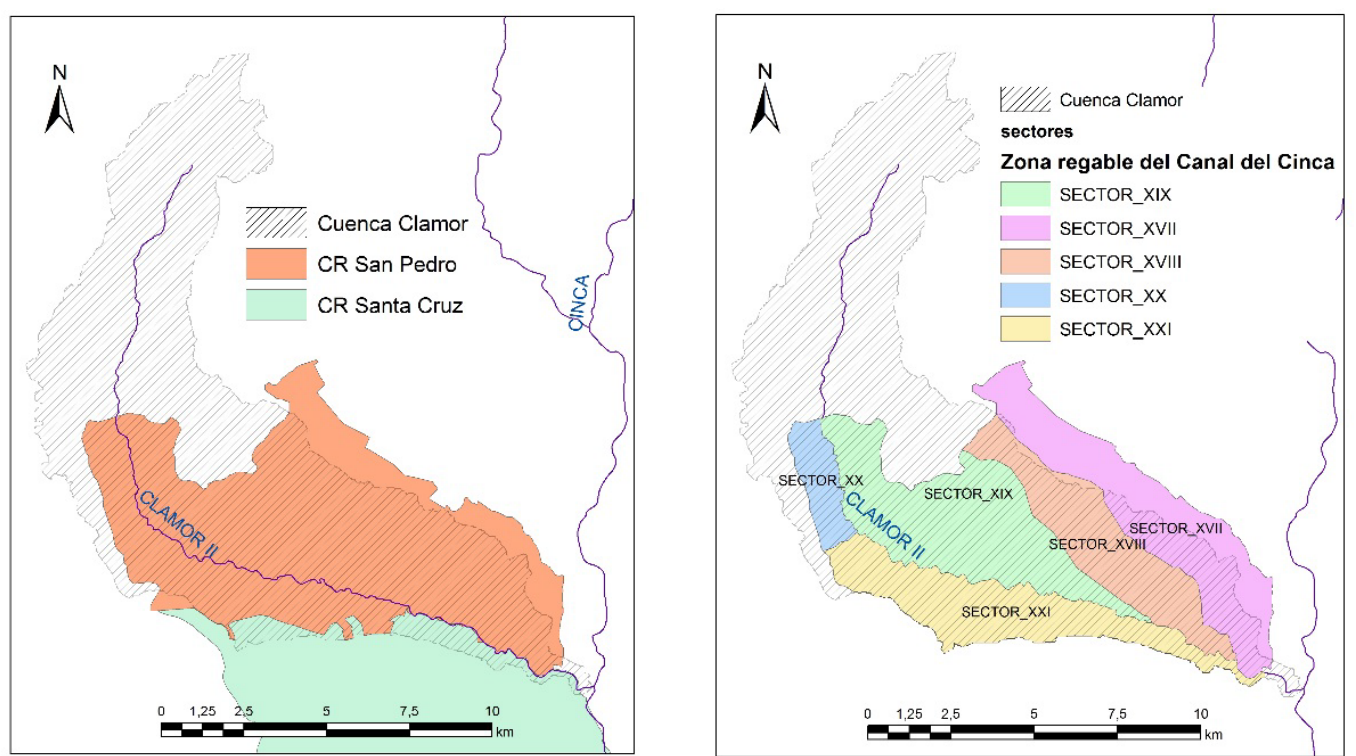

Figura 1. Ubicación de la cuenca y distribución de la superficie de riego por sectores

El marco geológico (Figura 2) son estratos horizontales arcillo-limosos con escasas intercalaciones de arenisca del Mioceno continental del valle del Ebro. Están muy erosionados, las zonas altas protegidas por mantos de grava pliocuaternarios (sasos), y el resto excavado en un valle de fondo plano que desemboca en las terrazas aluviales del Cinca. Se han descrito tres tipos de suelos: Xerorthent Típico, Xerofluvent Típico y Calcixerept Típico. Su granulometría es heterogénea, con representación de casi todas las clases texturales incluso limosa o arcillosa. El limo es predominantemente fino, sobre todo en las texturas franco-arcillo-limosas y arcillolimosa, donde el limo fino constituye alrededor del 50\% del total de la granulometría. Una parte importante de los suelos de esta cuenca está afectada por problemas de salinidad y sodicidad por lo que esta yerma o con cultivo de arroz. Su conductividad hidráulica es mayoritariamente 


\section{CONGRESO IBÉRICO DE AGROINGENIERÍA \\ X CONGRESSO IBÉRICO DE AGROENGENHARIA \\ 3 - 6 septiembre 2019, Huesca - España}

baja (0,05 a 0,3 m/día) o muy baja (menos de $0.05 \mathrm{~m} /$ día) a excepción de los suelos de texturas gruesas donde es media (0.3 a $1 \mathrm{~m} /$ día) [4].

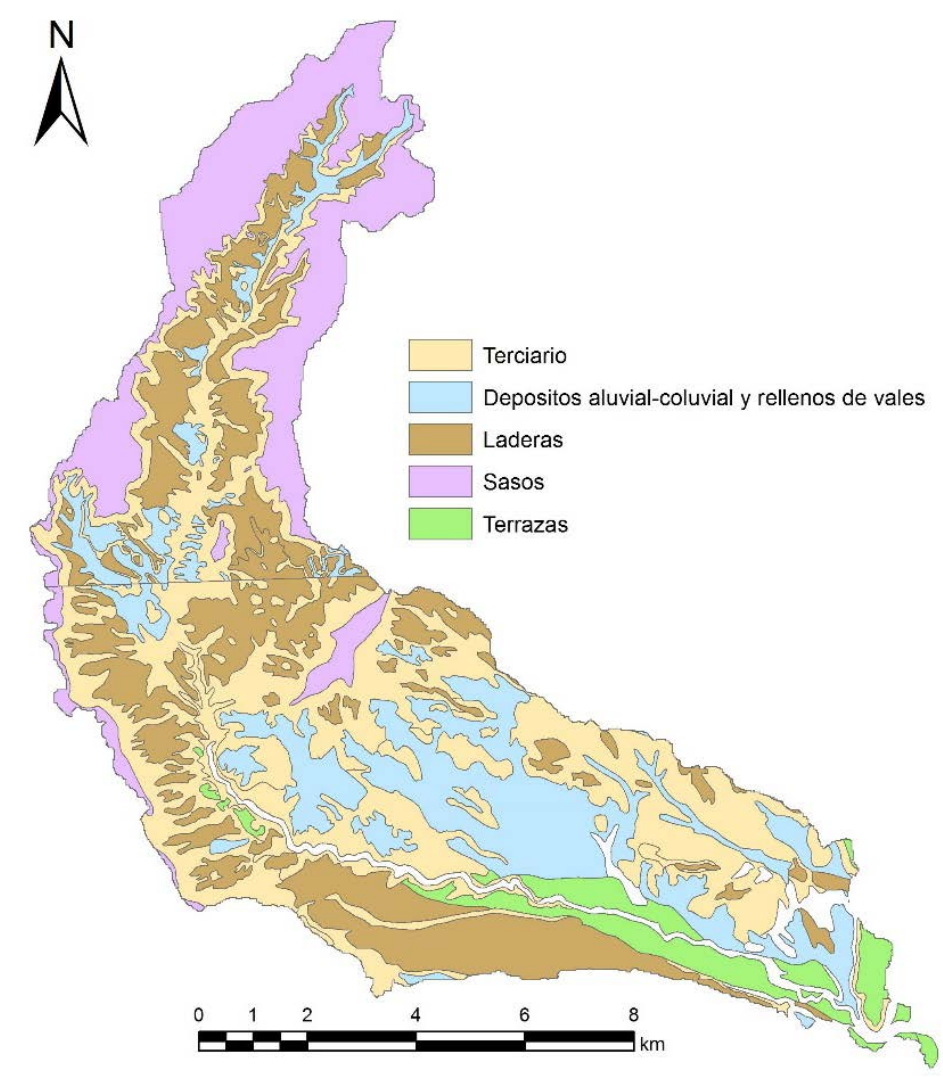

Figura 2. Geología de la cuenca de la Clamor Vieja

\section{Materiales y métodos}

\subsection{Uso del suelo, agua y nitrógeno}

La base cartográfica es el parcelario del SIGPAC. Los datos del cultivo por parcela se han obtenido a partir de las declaraciones de los agricultores para la PAC y por agregación, los mapas de cultivo. La ETP se calcula por Penman-Monteith. El agua aplicada se obtuvo de la comunidad de regantes contrastada con encuestas a regantes para determinar, además, prácticas agronómicas, fertilización, tipos de fertilizantes, fechas de aplicación y rendimiento de cultivos. La extracción de nitrógeno por los cultivos (NE) se obtuvo como el producto de su rendimiento por el contenido de $\mathrm{N}$ en la cosecha. El contenido de $\mathrm{N}$ en cada $1000 \mathrm{~kg}$ de materia seca cosechada se estimó: $22 \mathrm{~kg}$ para arroz, 24 kg para cebada, 17,3 kg para grano de maíz (14\% de humedad), $29 \mathrm{~kg}$ para alfalfa, $22 \mathrm{~kg}$ para el resto de los forrajes y $12,5 \mathrm{~kg}$ de guisantes. No se consideran las extracciones de $\mathrm{N}$ contenida en los restos de cosecha del maíz porque es usual incorporar los residuos de cosecha al suelo. Se analiza el manejo del riego mediante el rendimiento del riego o "Relative Irrigation Supply" (RIS) calculado como el coeficiente entre la cantidad de agua aplicada y las necesidades de riego netas (NHn). El manejo del abonado nitrogenado es la relación entre la fertilización nitrogenada mineral y la extracción de los cultivos (NFM/NE). 


\section{CONGRESO IBÉRICO DE AGROINGENIERÍA \\ X CONGRESSO IBÉRICO DE AGROENGENHARIA \\ 3 - 6 septiembre 2019, Huesca - España}

\subsection{Volumen y composición del drenaje}

El drenaje se aforó con un minimolinete OTT-C2, por el método de las secciones. Se muestreó con frecuencia semanal durante la campaña de riego y quincenal fuera esta. In situ se mide temperatura y conductividad eléctrica (CE) con un equipo 315i de WTW. Se determinó la concentración de nitrato en la EPS de Huesca mediante acidificación con $\mathrm{HCl} 1 \mathrm{~N}$ por espectrometría ultravioleta en un espectrofotómetro Unicam 5625 UV/VIS Spectrometer con lámpara de deuterio, a 220 y $275 \mathrm{~nm}$ en cubeta de cuarzo de $1 \mathrm{~cm}$ de recorrido de luz. Se determinaron las concentraciones de sodio, calcio y magnesio para calcular el SAR.

\subsection{Balance hidrológico de la cuenca}

Se han realizado dos balances hídricos a dos escalas diferentes y utilizando metodologías diferentes. El nivel básico, común en la agricultura, es el cultivo como única unidad de gestión. El segundo nivel, utilizado ampliamente en hidrología, es la cuenca. El balance de agua por cultivo permite la estimación del volumen de agua utilizada por todos los cultivos a través de la evapotranspiración (ET) y el volumen drenado por el suelo (DBAS). Utilizando los datos de riego se realiza un balance mensual de agua en el suelo para cada uno de los cultivos presentes en la cuenca entre abril del 2009 y septiembre del 2010. El balance siguiendo la metodología expuesta por [5], hace una distribución de la precipitación (P) y riego (R) entre la evapotranspiración (ET), las pérdidas por evaporación y arrastre del riego por aspersión (PEA) y DBAS. Las PEA se estimaron según [6]. Desconocida el agua almacenada en el suelo al inicio del estudio (1 de abril de 2009), se asume que el contenido de agua en esta fecha era igual a la mitad de la CRAD.

En una segunda etapa, se ha realizado el balance hidrológico de toda la cuenca que requiere sumar los datos de las parcelas incluidas en la cuenca más los del área de secano para conocer los valores totales de P, R, ET, y PEA. Para ello se necesita determinar dos parámetros más: el agua de canal sobrante vertida al barranco (C) y el drenaje real en el barranco de la Clamor Vieja (D). Así pues, el balance de agua se hizo con los volúmenes asociados a los flujos de entrada $(\mathrm{E}=\mathrm{P}+\mathrm{R}+\mathrm{C})$ y salida $(\mathrm{S}=\mathrm{ET}+\mathrm{D}+\mathrm{PEA})$ del sistema cuenca. Estos volúmenes se agruparon por periodos semestrales con riego (abril a septiembre) y sin riego (octubre a marzo). El error del balance se calcula en términos porcentuales como $200 \cdot[(E-S-A) /(E+S+A)]$, no admitiendo errores superiores al 10\% para los balances hidrológicos.

Para evaluar el uso del agua según un modelo de contabilidad del agua hay que determinar los diferentes usos que tienen los flujos de salida del sistema [7]. Por lo que se asigna a cada una de las salidas del balance su uso correspondiente:

- La evapotranspiración productiva (ETp) se corresponde con la ET de los cultivos.

- Se considera evapotranspiración no productiva (ETnp) a la ET de las superficies sin cultivo y a las PEA.

- La escorrentía/percolación se corresponde con el drenaje. Puede ser reutilizable (E/P r) o no reutilizable (E/P nr) dependiendo de la escala espacial que se aplique y de la calidad del agua de drenaje. Si se considera la escala espacial de la cuenca del Ebro, el agua de drenaje de la Clamor Vieja retorna al río Cinca y puede ser reutilizada. Por lo tanto, se consideraría como reutilizable.

Los indicadores hidrológicos relacionan entre sí los volúmenes de los usos del agua mediante las siguientes fracciones:

$\mathrm{FC}=(\mathrm{VETp}+\mathrm{VETnp}+\mathrm{VE} / \mathrm{Pnr}) / \mathrm{V}$

$\mathrm{FR}=\mathrm{VE} / \mathrm{Pr} / \mathrm{V}$

$\mathrm{FPC}=\mathrm{VETp} /(\mathrm{VETp}+\mathrm{VETnp}+\mathrm{VE} / \mathrm{Pnr})$

Donde FC es la fracción consuntiva (\%), VETp es el volumen de la evapotranspiración productiva, VETnp es el volumen de la evapotranspiración no productiva, VE/Pnr es el 
volumen de la escorrentía/percolación no recuperable, FR es la fracción recuperable (\%), VE/Pr es el volumen de la escorrentía/percolación recuperable, $\mathrm{V}$ es el volumen de agua total disponible, y FPC es la fracción productiva consuntiva (\%).

\subsection{Balance de sales}

En el balance de sales se calcula la masa de sales en cada componente del balance hídrico, realizado como el producto del volumen de agua y su concentración de sales (producto de la $\mathrm{CE}$ del agua en dS/m por el factor de conversión de $640 \mathrm{mg} / \mathrm{L}$ ). La evapotranspiración y a las pérdidas por evaporación y arrastre del riego por aspersión son los dos componentes de balance hídrico que no se tienen en cuenta por considerarse libres de iones. También se supone que la exportación de sales por las cosechas es insignificante. Así pues, el balance de sales tiene los siguientes componentes: entrada de sales por riego (SR) y precipitación (SP) y salida por drenaje (SD). El incremento de sales en el sistema formado por el regadío de la cuenca $(\Delta S)$ es la diferencia entre las entradas SR y SP y la salida SD. Si este $\Delta$ S es positivo se interpreta como procesos de acumulación de sales y si es negativo como lavado de sales fuera de la cuenca.

\subsection{Balance de nitrógeno}

Se ha realizado el balance de $\mathrm{N}$ entre noviembre de 2008 y octubre de 2010 considerando las entradas y salidas de $\mathrm{N}$ más importantes del sistema. Las pérdidas a la atmósfera por volatilización y por desnitrificación no se han tenido en cuenta, por carecer de datos. Las entradas consideradas son la masa de nitrógeno en el agua de riego (NR), en la precipitación (NP) y en el vertido del agua sobrante del canal al barranco (NC). Además, se ha considerado el nitrógeno aplicado como fertilizante mineral (NFM), el nitrógeno aplicado como fertilizante orgánico (NFO), y la fijación simbiótica por las leguminosas (NFS). Las salidas consideradas han sido la masa de nitrógeno en el agua de drenaje (ND), y la extracción por los cultivos (NE). La diferencia entre entradas y salidas es la variación de nitrógeno en el sistema $(\Delta N)$. El NFM se obtuvo multiplicando las dosis de abonado de las encuestas por la riqueza en $\mathrm{N}$ del abono utilizado. La alfalfa puede cubrir sus necesidades de $\mathrm{N}$ gracias a la fijación simbiótica. Si se le aporta abono nitrogenado, la planta toma con preferencia el aportado, deja de producir nódulos y de fijar nitrógeno atmosférico a través de las bacterias. A pesar de esto, los agricultores realizan habitualmente aportaciones nitrogenadas en forma mineral, aunque no cubren el total de las necesidades situadas en torno a los $400 \mathrm{~kg}$ N/ha. La fijación simbiótica se encarga de cubrir el resto de las necesidades de la alfalfa. Además, cuando se alza un alfalfar, la cantidad de $\mathrm{N}$ que queda en el suelo en las coronas y raíces dejadas en el campo, supone al menos $150 \mathrm{~kg}$ $\mathrm{N} / \mathrm{h}$. La liberalización de nitrógeno de coronas, raíces y restos de cosecha es lenta y se produce a lo largo de los tres o cuatro años siguientes al enterrado [8]. Para cuantificar este aporte de $\mathrm{N}$ por mineralización se toma un valor de $37,5 \mathrm{~kg} \mathrm{~N}$ por hectárea y se multiplica por la superficie de alfalfa levantada.

El nitrógeno aportado por fertilización orgánica (NFO) se calcula a partir del censo ganadero de la comunidad de regantes: 26.270 cerdos, 5.701 ovejas y 570 terneros. El valor neto de $\mathrm{N}$ que llega al suelo por tipo de animal y año se ha estimado en de $8,7 \mathrm{~kg}$ por cerdo; $18,9 \mathrm{~kg}$ por oveja y $77 \mathrm{~kg}$ por ternero [9]. Se supone que el purín y estiércol se aplica de forma homogénea en toda la superficie cultivada de la cuenca con un valor medio anual de $41 \mathrm{~kg}$ $\mathrm{N} / \mathrm{ha}$. La masa de $\mathrm{N}$ que entrada en el agua de lluvia (NP) y de riego (NR) se determinó como el producto del volumen por la concentración de nitrato de cada uno. La masa de nitrógeno exportado a través del barranco (ND), se calculó como el producto del volumen de agua aforado por su concentración de nitrógeno nítrico. Se asume que la mayor parte del $\mathrm{N}$ presente en el agua se encuentra en forma de nitrato. Finalmente, el incremento de $\mathrm{N}$ en el regadío de la 


\section{CONGRESO IBÉRICO DE AGROINGENIERÍA \\ X CONGRESSO IBÉRICO DE AGROENGENHARIA \\ 3- 6 septiembre 2019, Huesca - España}

cuenca se calcula como las entradas (NFM+NFO+NFS+NP+NR+NC) menos las salidas $(\mathrm{NE}+\mathrm{ND})$.

\section{Resultados y discusión}

\subsection{Cultivos}

La Figura 3 presenta los mapas de cultivo durante las campañas de 2009 y 2010. El secano supone el $46 \%$ de toda la cuenca, predominando el cultivo de cebada con 2.086 y 2.273 has en 2009 y 2010 respectivamente.

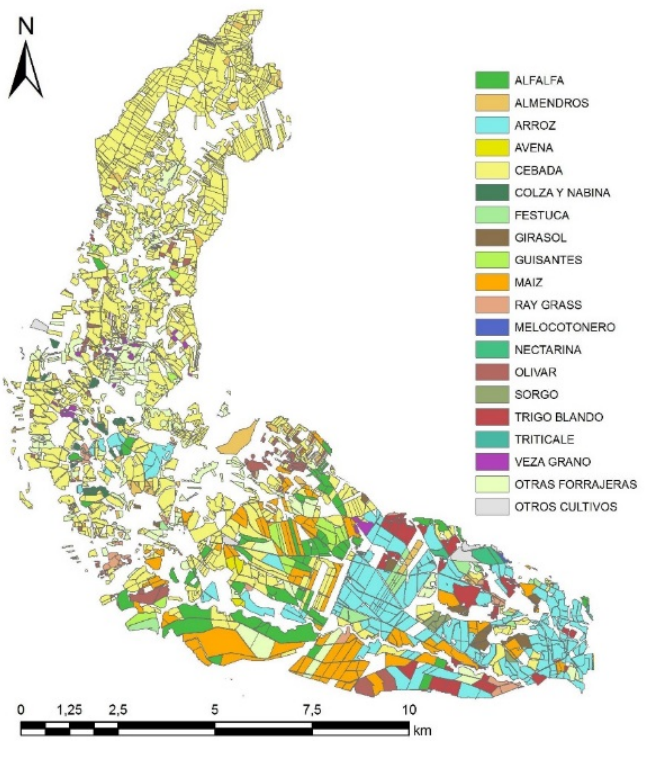

2009

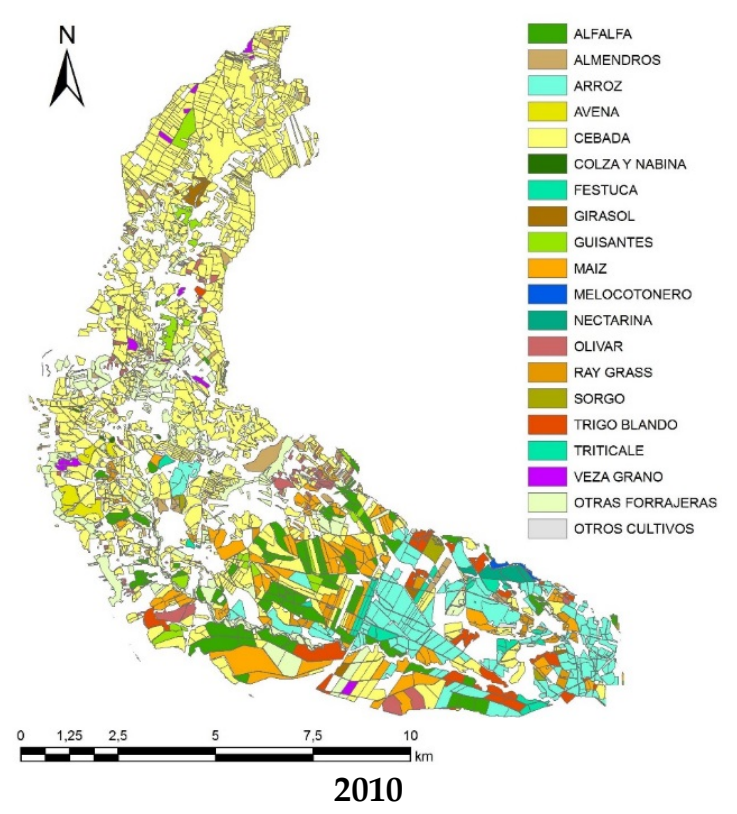

2010

Figura 3. Mapas de cultivo de la cuenca de la Clamor Vieja durante las campañas 2009 y 2010.

Los cultivos mayoritarios en regadío fueron arroz, maíz, cebada y alfalfa. La distribución de superficies fue similar en ambas campañas con un ligero descenso de arroz y maíz en 2010 respecto al 2009, y un aumento en cebada y alfalfa.

El arroz es el cultivo mayoritario, 22\% de la superficie de regadío cultivada. Fangueo en noviembre, paso de chisel y abonado de fondo en marzo seguido de pase con grada rotativa. En mayo, embalse y siembra con $200 \mathrm{~kg} / \mathrm{ha}$. Para cultivo sin fangueo, en febrero se hace un pase de grada. En junio, herbicida y un abonado de cobertera. Se cosecha en octubre, con rendimiento en torno a $6.000 \mathrm{~kg} / \mathrm{ha}$. El riego en esta zona oscila entre 900 y $1.400 \mathrm{~mm} / \mathrm{año}$. Es el cultivo más regado durante las dos campañas con un valor medio de $1080 \mathrm{~mm} / \mathrm{año}$.

En el $17 \%$ del regadío se cultivó maíz, que se siembra a principios de mayo, tras picado de rastrojo, pases de chisel y grada rotativa y abonado de fondo. La dosis media de siembra es de 85.000 plantas/ha y después se aplica un herbicida de pre-emergencia. Los riegos se hacen de julio a septiembre con un riego medio anual de $735 \mathrm{~mm}$. Se hacen dos abonados por fertirrigación y un tratamiento herbicida de post-emergencia. Hacia noviembre se cosechan, aproximadamente $14.000 \mathrm{~kg} / \mathrm{ha}$. En las segundas cosechas, tras guisante o cebada, las labores y fechas de siempre son ligeramente diferentes y una siembra más tardía. Tras guisante se obtienen unos $13.000 \mathrm{~kg} / \mathrm{ha}$. Tras cebada, $11.000 \mathrm{~kg} / \mathrm{ha}$.

En el $16 \%$ de la superficie regada hubo cebada. Si sigue a maíz se pican sus restos y se da un pase de chisel y luego subsolador y un abonado de fondo. Se siembran $200 \mathrm{~kg} / \mathrm{ha}$ en noviembre. En marzo se aplica herbicida, fungicida y un abonado de cobertera. La cebada 


\section{CONGRESO IBÉRICO DE AGROINGENIERÍA \\ X CONGRESSO IBÉRICO DE AGROENGENHARIA \\ 3- 6 septiembre 2019, Huesca - España}

recibe unos $165 \mathrm{~mm}$ de agua de riego siendo el cultivo menos regado. En junio se cosecha, 5.000 a $7.000 \mathrm{~kg} / \mathrm{ha}$ frente a los $2.500 \mathrm{~kg} / \mathrm{ha}$ de secano.

La alfalfa ocupa el 13\% del regadío. Se siembra habitualmente en otoño, aunque en algún caso en primavera. Antes de la siembra se pasan subsolador, cultivador, rulo, abonado de fondo y pase de la grada. Tras la siembra se hace un pase de molón. Cuando la alfalfa ya está implantada, del $2^{\underline{o}}$ año en adelante recibe dos o tres abonados de cobertera en abril, junio y agosto con dosis bajas de N. Normalmente la alfalfa se mantiene en el campo unos cinco años y recibe cinco o seis cortes al año, el primero a mitad de abril y el último en octubre, con un intervalo entre cortes de 35 días. El riego medio aplicado es de $680 \mathrm{~mm} / \mathrm{año}$. Los rendimientos anuales de alfalfa están en torno a $15.000 \mathrm{~kg} / \mathrm{ha}$.

\subsection{Uso del suelo, agua y nitrógeno}

La Tabla 1 resume los valores de superficie, riego, necesidades hídricas netas (NHn), índice RIS, dosis de fertilización nitrogenada mineral (NFM), nitrógeno extraído teóricamente por los cultivos (NE) y su relación (NFM/NE) para los cultivos más importantes en el regadío de la cuenca Clamor Vieja. Según el índice RIS, el exceso de riego en arroz, maíz y cebada fue del 50\% y del $20 \%$ en alfalfa. El mayor valor de NFM se da en el maíz con $263 \mathrm{~kg} / \mathrm{ha}$, similar al aplicado en otras áreas de riego por aspersión (Sexton et al 1996). El valor de NFM fue similar para arroz y cebada siendo de 165 y $163 \mathrm{~kg} /$ ha respectivamente. El valor de NFM de la cebada es similar al de otros regadíos por superficie [10] y por aspersión [11] del valle del Ebro. La relación entre la fertilización nitrogenada mineral y la extracción por cultivos (NFM/NE) varía entre el 0,3 de la alfalfa y el 1,2 del arroz. El valor de la alfalfa se explica porque se le aplica un $30 \%$ de sus necesidades nitrógeno y el resto se fija simbióticamente. Por el contrario, en el cultivo del arroz se produce un $20 \%$ de exceso de fertilización nitrogenada mineral. Este abono que no se extrae por el arroz podría estar disponible para el siguiente cultivo que también será arroz, pero el exceso de riego lava el nitrógeno sobrante que entra en el ciclo del agua contaminando el agua subterránea y la superficial de los colectores de drenaje. De esta manera las parcelas cultivadas con arroz se identifican como la principal fuente de contaminación por $\mathrm{N}$ causada por fertilización excesiva y exceso de riego.

Tabla 1. Superficie, riego, necesidades hídricas netas (NHn), índice RIS, fertilización nitrogenada mineral (NFM), extracción nitrogenada de los cultivos (NE) y su relación (NFM/NE) para los principales cultivos de regadío de la cuenca de la Clamor vieja durante las campañas de riego de 2009 y 2010.

\begin{tabular}{|c|c|c|c|c|c|c|c|c|c|c|c|}
\hline \multirow{3}{*}{ Cultivos } & \multirow{2}{*}{\multicolumn{2}{|c|}{$\frac{\text { Superficie }}{\text { ha }}$}} & \multirow{2}{*}{\multicolumn{2}{|c|}{$\begin{array}{c}\text { Riego } \\
\mathrm{mm}\end{array}$}} & \multirow{2}{*}{\multicolumn{2}{|c|}{$\frac{\mathrm{NHn}}{\mathrm{mm}}$}} & \multirow{2}{*}{\multicolumn{2}{|c|}{ RIS }} & $N_{F M}$ & $\mathrm{~N}_{\mathrm{E}}$ & \multirow{2}{*}{$\mathbf{N}_{\mathrm{FM}} / \mathrm{N}_{\mathrm{E}}$} \\
\hline & & & & & & & & & \multicolumn{2}{|c|}{ kg/ha } & \\
\hline & 2009 & 2010 & 2009 & 2010 & 2009 & 2010 & 2009 & 2010 & \multicolumn{3}{|c|}{ 2009-10 } \\
\hline Arroz & 1.034 & 896 & 980 & 1.180 & 700 & 773 & 1,4 & 1,5 & 165 & 132 & 1,2 \\
\hline Cebada & 622 & 777 & 120 & 210 & 79 & 144 & 1,5 & 1,5 & 163 & 144 & 1,1 \\
\hline Maíz & 770 & 701 & 670 & 800 & 428 & 533 & 1,6 & 1,5 & 263 & 242 & 1,1 \\
\hline Alfalfa & 485 & 633 & 620 & 740 & 495 & 601 & 1,3 & 1,2 & 142 & 435 & 0,3 \\
\hline
\end{tabular}

\subsection{Cantidad y calidad del agua drenada}

La Figura 4 muestra la evolución del caudal (Q), conductividad eléctrica (CE) y concentración de nitratos [ $\left.\mathrm{NO}_{3}^{-}\right]$en el barranco de la Clamor Vieja entre abril de 2009 y noviembre de 2010. Durante las campañas de riego el caudal de drenaje aumenta siendo el volumen de agua drenado seis veces superior al de fuera de la campaña de riego. El régimen hídrico de la cuenca está regulado esencialmente por el riego como ocurre en otras muchas zonas semiáridas con regadío [12,13]. El mayor pico de drenaje fue en agosto de 2009, mes en el 


\section{CONGRESO IBÉRICO DE AGROINGENIERÍA \\ X CONGRESSO IBÉRICO DE AGROENGENHARIA \\ 3 - 6 septiembre 2019, Huesca - España}

que se registraron elevadas precipitaciones. La CE del agua de drenaje osciló entre 0,48dS/m, y $3,47 \mathrm{dS} / \mathrm{m}$, registrándose los menores valores durante las campañas de riego y los máximos fuera de ella. En general, los valores más elevados de concentración de nitratos, se observaron durante los meses de invierno. Las concentraciones de nitrato presentaron valores bajos estando todas las muestras por debajo de los $30 \mathrm{mg} / \mathrm{l}$. Se aprecia una evolución temporal conjunta de la concentración del nitrato y de la conductividad eléctrica. Durante la campaña de riego estos valores se reducen a la mitad en el caso de $\left[\mathrm{NO}_{3}{ }^{-}\right]$y un $60 \%$ en el caso de la $\mathrm{CE}$ por el efecto de la dilución que producen los retornos de riego.

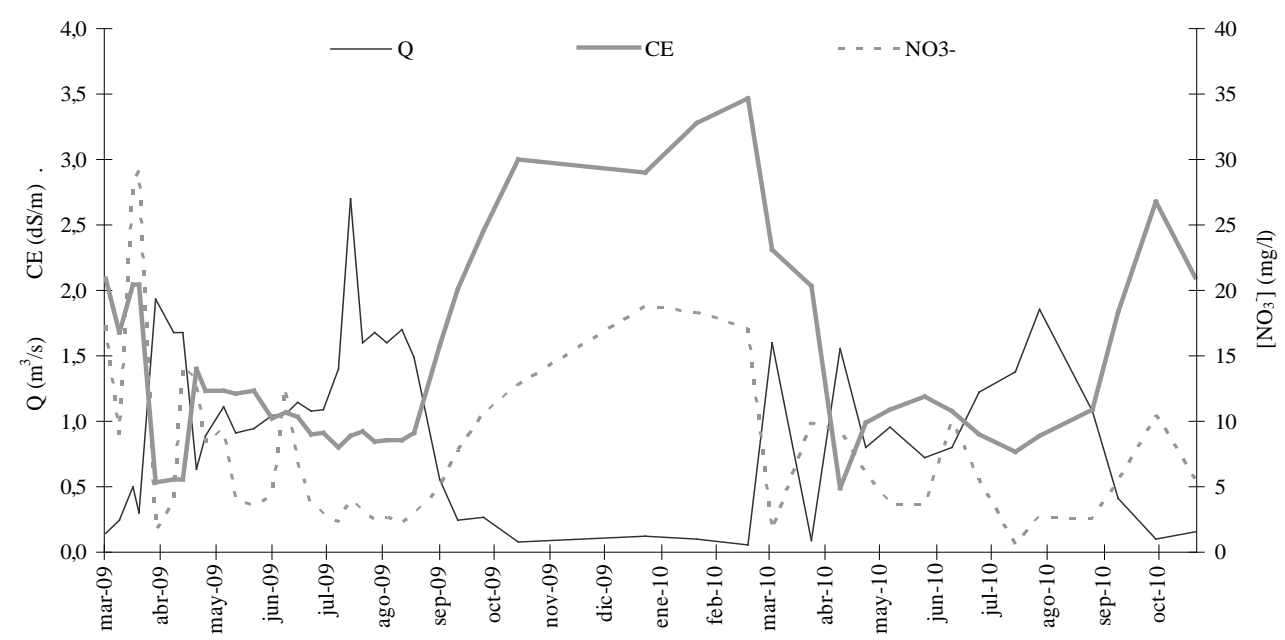

Figura 4. Evolución del caudal $(\mathrm{Q})$, conductividad eléctrica (CE) y concentración de nitratos $\left[\mathrm{NO}_{3}{ }^{-}\right]$en el barranco de la Clamor Vieja entre abril del 2009 y octubre del 2010.

\subsection{Balance de agua}

La evolución mensual del balance de agua en la cuenca muestra que durante las campañas de riego el valor de las entradas y salidas se duplica, considerando toda la cuenca y se triplica si se computa sólo el regadío (Figura 4). El riego (R) es la principal entrada de agua a la cuenca durante la campaña de riego, de abril a octubre. El riego varía lógicamente a lo largo de la campaña de riego y alcanza sus mayores valores mensuales en los meses de julio y agosto situándose en torno a los 150 $\mathrm{mm}$. En cuanto a las salidas la más importante fue la evapotranspiración (ET) con un valor medio de $650 \mathrm{~mm}$ para el regadío, durante la campaña de riego.

El agua drenada por la cuenca durante las campañas de riego tuvo un valor medio de 157 $\mathrm{mm}$, lo que constituye el 30\% de las salidas. La evolución del drenaje es igual durante las dos campañas de riego ya que en ambas tiene dos picos, en mayo y agosto. Si se considera que el agua de drenaje proviene solo de la superficie de regadío el volumen drenado durante la época de riego supera los $400 \mathrm{~mm}$. Este valor tan alto es propio de zonas de riego por superficie $[14,15]$. El riego influye en el incremento de los volúmenes de salida de agua por evapotranspiración y sobre todo en el drenaje.

El balance de agua de la cuenca se presenta en la Tabla 2. El cierre de los balances de agua durante las dos campañas de riego tiene errores ligeramente superiores al $10 \%$, pero el error acumulado de los 18 meses es sólo del 1,1\% a favor de las entradas, por lo que el balance de agua se considera correcto. La precipitación (P) es la principal entrada de agua a la cuenca durante todos los periodos a excepción de la campaña de riego del 2010 donde lo es el riego (R) seguido del vertido de agua del canal (C). La evapotranspiración (ET) constituye el 72\% de la salida de agua seguida del drenaje (D) con un $26 \%$ y las PEA con un $2 \%$. El drenaje es seis veces superior durante las épocas de riego frente a al periodo sin riego. 


\section{CONGRESO IBÉRICO DE AGROINGENIERÍA \\ X CONGRESSO IBÉRICO DE AGROENGENHARIA \\ 3 - 6 septiembre 2019, Huesca - España}

La entrada media anual de agua a la cuenca es de $790 \mathrm{~mm}$ de los que 415 son por precipitación, 237 por riego y 138 por vertido del canal. La Figura 6 muestra el reparto que se ha hecho del agua disponible por parte de los regantes entre los posibles usos según un modelo contable. En la cuenca se consume una media de $597 \mathrm{~mm}$ de agua al año, de los que $371 \mathrm{~mm}$ se han evapotranspirado por los cultivos. Por el barranco de la Clamor Vieja retornan al río Cinca $184 \mathrm{~mm}$ de agua. Dicho de otro modo, la fracción consuntiva (FC) se sitúa en el 76 \% y la recuperable (FR) en el 23\% respecto al agua total de entrada. Debido al error del balance, las fracciones calculadas suman $99 \%$ en vez de $100 \%$. La fracción consuntiva productiva (FCp) es del $62 \%$ y la consuntiva no productiva (FCnp) es del $38 \%$.

En la zona de regadío, R + P es igual a $1033 \mathrm{~mm}$, de los que $925 \mathrm{~mm}$ se consumen por ET. Aunque sólo $671 \mathrm{~mm}$ corresponden a la evapotranspiración directa de los cultivos. Según el balance de agua en el suelo, el $10 \%$ de las entradas abandona la zona radicular y se convierte en drenaje (Ds), con unos valores del 20\% para el arroz, $2 \%$ para la cebada, $12 \%$ para el maíz y $0 \%$ para la alfalfa. Para toda la cuenca se estima un valor de DS equivalente a $41 \mathrm{~mm}$, este valor es próximo los $47 \mathrm{~mm}$, resultantes del restarle los $138 \mathrm{~mm}$ de aportación directa del canal (C) a los $184 \mathrm{~mm}$ del drenaje real (D).

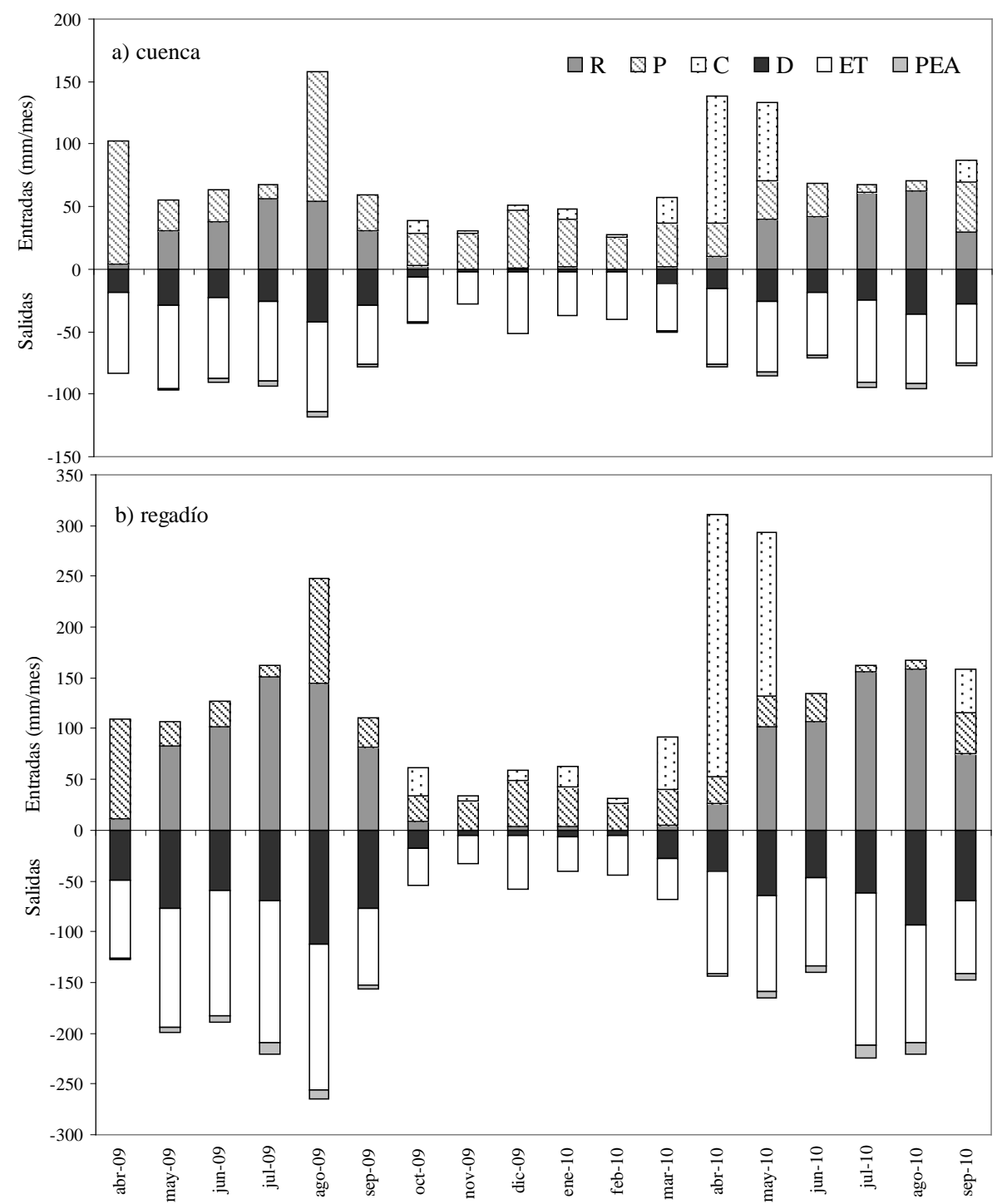

Figura 5. Evolución mensual de las entradas (R, P, C) y salidas (ET, D, PEA) de agua en la cuenca a) y en el regadío b) entre abril de 2009 y octubre de 2010. 


\section{CONGRESO IBÉRICO DE AGROINGENIERÍA \\ X CONGRESSO IBÉRICO DE AGROENGENHARIA \\ 3 - 6 septiembre 2019, Huesca - España}

Tabla 2. Entradas (E) [precipitación (P), riego (R), vertido del canal al barranco (C)], Salidas (S) [evapotranspiración (ET), drenaje (D), perdidas por evaporación y arrastre del riego por aspersión (PEA)] y error del balance de agua en la cuenca de la Clamor Vieja entre abril de 2009 y septiembre de 2010 .

\begin{tabular}{cccccc}
\hline & & $\begin{array}{c}\text { Abril/ } \\
\text { septiembre 2009 }\end{array}$ & $\begin{array}{c}\text { Octubre 2009/ } \\
\text { marzo 2010 }\end{array}$ & $\begin{array}{c}\text { Abril/ } \\
\text { septiembre 2010 }\end{array}$ & $\begin{array}{c}\text { Abril 2009/ } \\
\text { septiembre 2010 }\end{array}$ \\
\cline { 3 - 6 } & & \multicolumn{5}{c}{ hm $^{3}$} \\
& $\mathrm{P}$ & 33,5 & 22,8 & 15,9 & 72,2 \\
& $\mathrm{R}$ & 24,4 & 0,9 & 28,1 & 53,5 \\
Entradas & $\mathrm{C}$ & 0 & 5,3 & 20,8 & 26,2 \\
& $\mathrm{ET}$ & 58,0 & 29,0 & 64,8 & 151,8 \\
\hline & $\mathrm{PEA}$ & 1,5 & 25,5 & 38,5 & 107,5 \\
Salidas & $\mathrm{D}$ & 19,0 & 0,1 & 2,0 & 3,6 \\
\hline Error Balance & $(\%)$ & $-10,2$ & 3,1 & 17,0 & 39,1 \\
Error Balance & $(\%)$ & $-10,2$ & 1,5 & 57,5 & 150,2 \\
acumulado & $(\%)$ & $-6,4$ & 12,1 & 1,1 \\
\hline
\end{tabular}

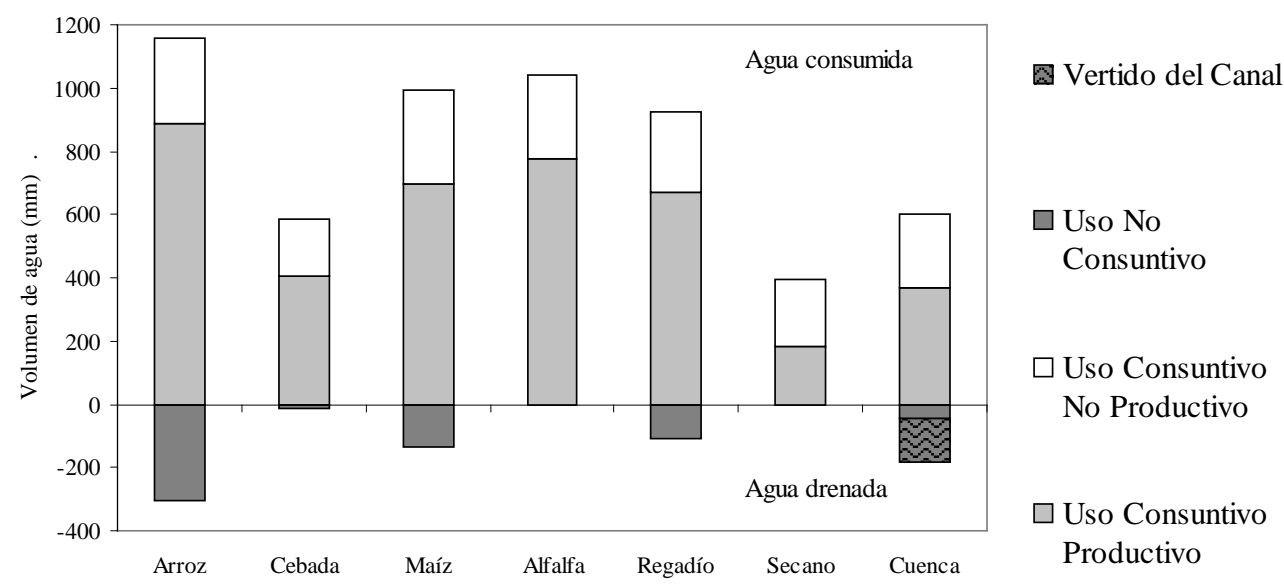

Figura 6. Usos del agua en la cuenca de la Clamor Vieja entre abril de 2009 y octubre de 2010.

\subsection{Balance de Sales}

Una vez realizado el balance de agua es posible proceder con el balance de sales. Como según éste en el secano no se produce drenaje, se asigna toda el agua drenada al regadío del cual se hace el balance de sales. La masa de sales se calcula a partir de la CE de algunos términos del balance de agua. La Tabla 3 resume el balance de sales y los valores de entradas y salidas de sales al regadío de la cuenca. Por cada ha de regadío entra una media anual de 1187 $\mathrm{kg}$ de sal en el agua de riego lo que supone el $62 \%$ de las entradas, a pesar de que el agua de riego tiene una baja concentración de sales. La siguiente no es una entrada como el resto, porque se vierte directamente al barranco, pero hay que contabilizarla. Como la evaporación y la evapotranspiración no exportan sales, la única salida es el drenaje y su valor medio anual es de $3878 \mathrm{~kg} / \mathrm{ha}$. Este valor de sales exportadas es similar al registrado en los regadíos de Bárdenas $[14,16,17]$ y entre tres y seis veces inferior a los registrados en el regadío de la cuenca de la Violada [10,18,19] y la cuenca de la Valcuerna [20]. Esta diferencia está relacionada con la presencia de yeso en el subsuelo de la cuenca de la Violada y Valcuerna. 


\section{CONGRESO IBÉRICO DE AGROINGENIERÍA \\ X CONGRESSO IBÉRICO DE AGROENGENHARIA \\ 3 - 6 septiembre 2019, Huesca - España}

Un 51\% de las sales exportadas por el drenaje procedió del lavado de las sales del suelo y disolución de los materiales geológicos, otro $31 \%$ tuvo su origen en las sales introducidas con el agua de riego y tan solo el $2 \%$ se correspondieron a las sales contenidas en el agua de lluvia. El otro $17 \%$ de las sales presentes en el agua de drenaje provienen del vertido de agua sobrante del canal de Terreu al barranco de la Clamor Vieja. La diferencia entre entradas y salidas de sales en el regadío de la Clamor Vieja fue negativa. Esto supone un lavado de las sales en torno a los $2.000 \mathrm{~kg} / \mathrm{ha}$ que puede asociarse principalmente al predominio de los procesos de disolución de sales sobre los de precipitación.

Tabla 3. Balance de sales: Entradas de sal [en la precipitación (SP), en el riego (SR), en el vertido del canal al barranco (SC)], Salida de sal [en el drenaje (SD),] e incremento de sales (๑S) en el regadío de la cuenca Clamor Vieja entre abril de 2009 y septiembre de 2010.

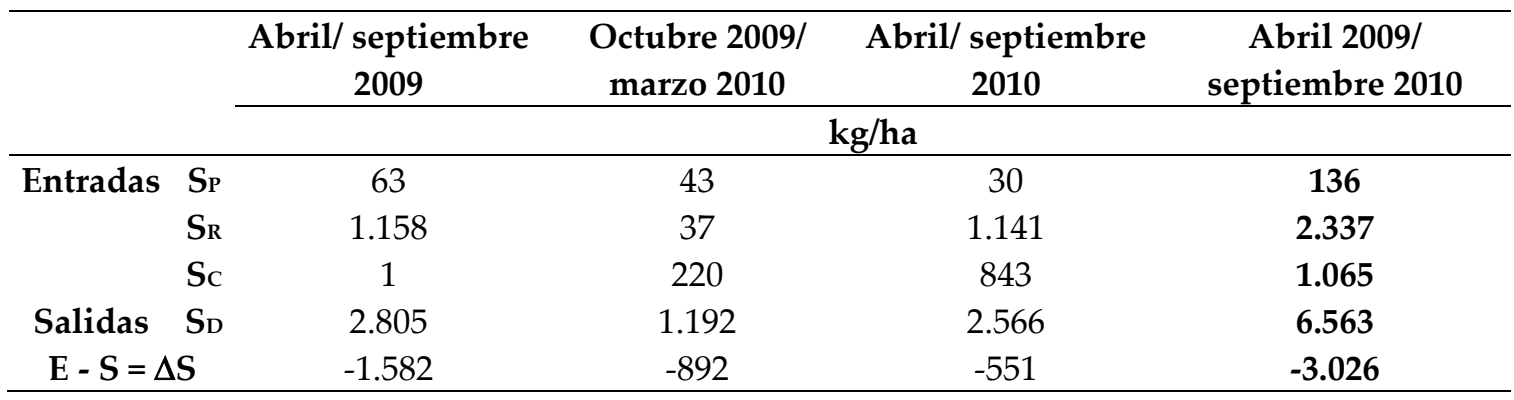

\subsection{Balance de nitrógeno}

El balance de nitrógeno se ha realizado basándose también en el balance hídrico entre abril de 2009 y septiembre de 2010. Es más complejo que el balance de sales debido a que las principales entradas y salidas, como fertilización y cosecha, dependen del manejo de cada agricultor.

La Tabla 4 muestra los principales componentes del balance de nitrógeno del regadío. Lluvia y el riego contribuyen anualmente con una media de con 5 y $2 \mathrm{~kg} \mathrm{~N} / \mathrm{ha}$ respectivamente. La fertilización mineral media es $191 \mathrm{~kg} \mathrm{~N} /$ ha y la orgánica $41 \mathrm{~kg} \mathrm{~N} / \mathrm{ha}$. La mineralización de las raíces y coronas de antiguos campos de alfalfa produce un valor de fijación simbiótica de la alfalfa de $1 \mathrm{~kg} \mathrm{~N} / \mathrm{ha}$. La fertilización mineral representa la principal entrada de $\mathrm{N}$ al sistema formado por el regadío (80\%). La siguiente entrada más importante sería la fertilización orgánica que representa el $17 \%$ de las entradas. El nitrógeno extraído por los cultivos del regadío (NE) supone el 97\% de las salidas y su valor medio es de $199 \mathrm{~kg} \mathrm{~N} / \mathrm{ha}$. El nitrógeno exportado por el agua de drenaje es la otra salida con un valor medio anual de $7 \mathrm{~kg} \mathrm{~N} / \mathrm{ha}$. Este valor es muy inferior al registrado en otras cuencas con riego por aspersión del valle del Ebro [21]. Según el balance de agua planteado los cultivos fertilizados y regados en exceso, como el arroz y el maíz, serían los responsables de la mayor parte de la exportación de nitrógeno. Sin embargo, el $\mathrm{N}$ exportado por el drenaje es sólo el 2,4\% de la fertilización orgánica y mineral. Este porcentaje resulta muy bajo respecto a otros estudios, y constata que los suelos poco permeables presentes en esta cuenca, dificultan el lavado de nitratos. La cantidad total de nitrógeno exportado por los cultivos fue de unos $199 \mathrm{~kg} \mathrm{~N} / \mathrm{ha}$. El abonado mineral fue de $191 \mathrm{~kg}$ $\mathrm{N} / \mathrm{ha}$, ajustándose a las extracciones. De este modo, cabe pensar que el $\mathrm{N}$ de la fertilización orgánica (41 kg N/ha), no es tenido en cuenta por los agricultores como un aporte efectivo de nitrógeno. 
X CONGRESO IBÉRICO DE AGROINGENIERÍA

X CONGRESSO IBÉRICO DE AGROENGENHARIA

3 - 6 septiembre 2019, Huesca - España

Tabla 4. Balance de nitrógeno. Nitrógeno total aportado y extraído ( $\mathrm{kg} / \mathrm{ha})$ en cada una de las cuencas estudiadas.

\begin{tabular}{|c|c|c|c|c|c|}
\hline & $\begin{array}{c}\text { Abril/ } \\
\text { septiembre } 2009\end{array}$ & $\begin{array}{c}\text { Octubre 2009/ } \\
\text { septiembre } 2010\end{array}$ & $\begin{array}{c}\text { Abril 2009/ } \\
\text { septiembre 2010 }\end{array}$ & $\begin{array}{c}\text { Abril 2009/ } \\
\text { septiembre 2010 }\end{array}$ \\
\hline & & & $\mathrm{kg} / \mathrm{ha}$ & & $\%$ \\
\hline \multirow{6}{*}{ E } & $\mathrm{N}_{\mathrm{P}}$ & 3,7 & 4,3 & 8,0 & 1,7 \\
\hline & $\mathrm{N}_{\mathrm{R}}$ & 2,8 & 1,7 & 4,5 & 0,9 \\
\hline & $\mathrm{N}_{\mathrm{FM}}$ & 193 & 190 & 383 & 80 \\
\hline & $\mathrm{N}_{\mathrm{FO}}$ & 43 & 39 & 82 & 17 \\
\hline & $\mathrm{N}_{\mathrm{FS}}$ & 0,9 & 1,1 & 1,9 & 0,4 \\
\hline & $\mathbf{N c}_{\mathrm{c}}$ & 0,0 & 1,6 & 1,6 & 0,3 \\
\hline \multirow{2}{*}{$S$} & $\mathrm{Ne}_{\mathrm{E}}$ & 197 & 200 & 397 & 97 \\
\hline & $\mathrm{N}_{D}$ & 6 & 6 & 11 & 3 \\
\hline \multicolumn{2}{|c|}{$E-S=\Delta N$} & 40,8 & 31,4 & 72,2 & \\
\hline
\end{tabular}

\section{Conclusiones}

La cuenca de la Clamor Vieja es una unidad hidrológica, básicamente arcillosa y muy interesante para estudiar el efecto de la transformación de riego a pie hacia riego por aspersión.

El drenaje de la Clamor vieja está directamente relacionado con el riego. No hay un acuífero amortiguador como en LASESA. Durante las campañas de riego el caudal de drenaje aumenta considerablemente siendo el volumen de agua drenado seis veces superior al de fuera de la campaña de riego.

La conductividad eléctrica del agua de drenaje osciló entre 0,48dS/m, y 3,47dS/m, registrándose los valores más bajos durante las campañas de riego y los máximos fuera de ella. En general, los valores más elevados de concentración de nitratos, se observaron durante los meses de invierno. La concentración de nitrato se encuentra siempre por debajo de los $30 \mathrm{mg} / \mathrm{l}$. Se aprecia una evolución temporal conjunta de la concentración del nitrato y de la conductividad eléctrica. Durante la campaña de riego estos valores se reducen a la mitad en el caso de nitrato y un $60 \%$ en el caso de la conductividad eléctrica por el efecto de la dilución que producen los retornos de riego.

El balance hídrico ha permitido conocer que la Fracción Consuntiva se sitúa en el 76 \% y la Recuperable en el $23 \%$ respecto al agua total de entrada. Debido al error del balance, las fracciones calculadas suman 99\% en vez de 100\%. La Fracción Consuntiva Productiva es del $62 \%$ y la Consuntiva No Productiva es del $38 \%$.

\section{Agradecimientos}

Agradecer la colaboración de la Comunidad de Regantes de San Pedro de Castelflorite y a su presidente, Alberto Loscertales, por la información aportada. Así mismo la ayuda de J.A. Manso de la EPSH.

\section{Referencias}

1. R. Andrés, J.A. Cuchí. Analysis of sprinkler irrigation management in the LASESA district, Monegros (Spain). Agricultural Water Management. 2014, 131: 95-107

2. Andrés, J.A. Cuchí. Salt and nitrate exports from the sprinkler irrigated Malfarás Creek Watershed (Ebro River Valley, Spain) during 2010. Environmental Earth Sciences. 2014, 72 (7), 2667-2682.

3. R. Andrés, J.A. Cuchí. The use of nitrogen in a sprinkler-irrigated district in Monegros (Northeast Spain). Agricultural Water Management.2014, 144:120-133.

4. R. Andrés, R. Proyecto de la red de drenajes para 1200 hectáreas de los sectores XVIII y XIX del Cinca. C.R. San Pedro (Castelflorite, Huesca). 2005. PFC. U. de Zaragoza. 26 p y anejos. 


\section{CONGRESO IBÉRICO DE AGROINGENIERÍA \\ $X$ CONGRESSO IBÉRICO DE AGROENGENHARIA \\ 3 - 6 septiembre 2019, Huesca - España}

5. J. Causapé. EMR 1.0: Herramienta al servicio de la vigilancia agroambiental del regadío. Información Técnica Económica Agraria. 2008, 104, 369-380.

6. E. Playán, R. Salvador, J.M. Faci, N., Zapata, A. Martínez-Cob, I. Sánchez. Day and night wind drift and evaporation losses in sprinkler solid-sets and moving laterals. Agricultural Water Management. 2005, 76(3), 139-159.

7. D.J. Molden. Accounting for water use and productivity. SWIM Paper 1. IWMI. Colombo, Sri Lanka. 1997. 16pp.

8. Delgado, F. Muñoz, D Andueza, N. Martínez. Acción del abonado nitrogenado sobre el crecimiento y la movilización de reservas nitrogenadas de la alfalfa. En Producción de pastos, forrajes y céspedes. Actas XLII Reunión Científica de la Sociedad Española para el Estudio de los Pastos 6-10 de mayo de 2002, Lérida (España) (pp. 327-332). Ediciones de la Universidad de Lérida.

9. F. Orús. Estiércoles, nitrógeno y cargas ganaderas. Criterios para la valoración del contenido de nitrógeno de los estiércoles, según la Unión Europea. Información Técnica, Gobierno de Aragón. 123. 2003. 16pp.

10. D. Isidoro, D. Quílez, R. Aragüés. Environmental impact of irrigation in La Violada district (Spain). Journal of environmental quality. 2006, 35(3), 776-785.

11. Cavero, A., Beltrán, R. Aragüés. Nitrate exported in drainage waters of two sprinkler-irrigated watersheds. Journal of Environmental quality. 2003, 32(3), 916-926.

12. X. Cai, D.C. McKinney, M.W. Rosegrant. Sustainability analysis for irrigation water management in the Aral Sea region. Agricultural systems. 2003, 76(3), 1043-1066.

13. G. Schoups, C.L. Addams, S. M. Gorelick. Multi-objective calibration of a surface water-groundwater flow model in an irrigated agricultural region: Yaqui Valley, Sonora, Mexico.Hydrology and Earth System Sciences Discussions. 2005. 2(5), 2061-2109.

14. J. Causapé, D. Quílez, R. Aragüés. Assessment of irrigation and environmental quality at the hydrological basin level: II. Salt and nitrate loads in irrigation return flows. Agricultural Water Management. 2004, 70(3), 211-228.

15. D. Isidoro, D. Quílez, R. Aragüés. Water balance and irrigation performance analysis: La Violada irrigation district (Spain) as a case study. Agricultural Water Management. 2004, 64(2), 123-142.

16. J. Causapé. Aprovechamiento del agua en los regadíos de Bardenas. Información Técnica Económica Agraria. 2009, 105(3), 202-221.

17. R. Abrahao, J. Causapé, I. García-Garizábal, D. Merchán, D. Implementing irrigation: Salt and nitrate exported from the Lerma basin (Spain). Agricultural Water Management. 2011, 102(1), 105-112.

18. J. Faci, R. Aragüeś, F. Alberto, D. Quilez, J. Machin, J.L. Arrue. Water and salt balance in an irrigated area of the Ebro River Basin (Spain). Irrigation Science. 1985, 6(1), 29-37.

19. R. Barros, D. Isidoro, R. Aragüés. Three study decades on irrigation performance and salt concentrations and loads in the irrigation return flows of La Violada irrigation district (Spain). Agriculture, ecosystems \& environment. 2012, 151, 44-52

20. Tedeschi, A. Beltran, R. Aragüés. Irrigation management and hydrosalinity balance in a semi-arid area of the middle Ebro river basin (Spain). Agricultural water management. 2001, 49(1), 31-50.

21. J. Cavero, A. Beltrán, R. Aragüés. (2003). Nitrate exported in drainage waters of two sprinklerirrigated watersheds. Journal of Environmental quality. 2003, 32(3), 916-926. 